\title{
Maintaining Gateway Connectivity in Multi-hop Ad hoc Networks
}

\author{
Robert Brännström ${ }^{1}$, Christer Åhlund ${ }^{2}$, and Arkady Zaslavsky ${ }^{3}$ \\ ${ }^{1}$ Department of Computer Science, Luleå University of Technology, SE-971 87 Luleå, Sweden \\ ${ }^{2}$ Division of Mobile Networking \& Computing, Luleå University of Technology, \\ SE-931 87 Skellefteå, Sweden \\ ${ }^{3}$ School of Computer Science \& Software Engineering, Monash University, \\ 900 Dandenong Road, Caulfield East, Vic 3145, Melbourne, Australia \\ E-mail: \{robert.brannstrom, christer.ahlund\}@ltu.se, a.zaslavsky@csse.monash.edu.au
}

\begin{abstract}
The need for maintaining gateway connectivity in an ad hoc access network is vital considering the 80/20 ratio of Internet traffic. There are several proposals of how to integrate gateway forwarding strategies but they all rely on the route discovery procedure of reactive routing protocols. We propose a proactive approach to avoid the delay of the route discovery process. Mobile IP is often suggested to handle macro mobility and we use the advertisements periodically sent by the gateway to update routing tables in the ad hoc network. Since advertisements may arrive to a mobile host through multiple paths, it is important to keep track of the best path to each gateway. We demonstrate the use of a proposed dynamic metric and how to handle location of correspondent hosts. A simulation study demonstrates the usefulness and efficiency of our approach.
\end{abstract}

\section{Introduction}

The advent of high bandwidth wireless networks [1]-[3] requires support for extended network protocols. Today wireless network access is provided by connecting to one access point (AP) at a time.

New functionality needs to be added to mobile hosts (MH) and wireless access networks to enable networking software to fully utilize the features and opportunities that come with wireless network access. Only then will MHs truly benefit from the dynamic behavior of wireless communications.

Global connectivity is achieved by the layering in the TCP/IP stack. In the physical layer, different physical equipment may be used, and in the data-link layer, different protocols can be used (e.g. Ethernet, Token Ring, Frame Relay). The network layer manages different data-link layer protocols and enables connectivity between them. The layers above the network layer (transport and application layer) are unaware of the differences in networking technologies, thus enabling global connectivity. When connecting ad hoc networks with wired IP networks, the differences between the two types of networks should be considered in the network layer too. Ad hoc networks are seen as a "none broadcast multiple access technology" (NBMA) [4] which requires new functionality at network layer. With the extended coverage that is achieved with multi-hop ad hoc networks connecting to a wired infrastructure, there is a high probability that MHs will discover multiple gateways. In this environment an $\mathrm{MH}$ should be able to use the best available gateway to communicate with a correspondent host and perhaps use multiple gateways for different hosts.

This paper proposes solutions towards enabling and supporting global connectivity in wireless ad hoc networks. In the proposed solutions the network layer software will evaluate and decide which wireless network connections to use. We describe the use of the Running Variance Metric (RVM) [5] and Relative Network Load (RNL) as performance metrics to classify the traffic load of gateways in wireless access networks. RVM and RNL can be efficiently used for infrastructure networks and ad hoc networks. In this paper we also use an extension to Mobile IP (MIP) [6] in order to enable mobile hosts to use multiple care-of addresses simultaneously [7]. The extension enhances network connectivity by enabling the mobile host, the home agent and correspondent hosts to evaluate and 
select the best connection. The proposed extension to Mobile IP is called Multihomed Mobile IP (M-MIP) to emphasize support for multiple connections for a mobile host at the same time. We describe a gateway architecture that integrates wired IP networks with ad hoc networks. Routes between a mobile host and gateways are maintained continuously where multi hop ad hoc connections are supported. Communication between peers in ad hoc networks is based on reactive ad hoc routing [8]. Mobile hosts moving between ad hoc networks are supported by Multihomed Mobile IP. We describe simulation results to validate the gateway selection strategy.

The rest of the paper is structured in the following way. Section 2 describes the formal reasoning used in the Global Connectivity solution and the gateway selection strategy. Section 3 describes a simulation model and the results of the simulation. Section 4 describes related works and section 5 concludes the paper.

\section{Global Connectivity}

MIP is used to manage MHs disconnecting from the home ad hoc network and connecting to foreign networks. MIP is extended to operate in ad hoc networks using a reactive routing protocol, where MIP messages are managed multiple hops instead of one hop as in the MIP specification. This enables MHs to register even if multiple hops from a gateway in the ad hoc network. The AODV protocol is modified to enable redistribution of MIP information and to create ad hoc routes based on MIP messages.

Since the $\mathrm{MH}$ is not associated when selecting which gateway to register with, the MH only has the knowledge from the agent advertisements. To evaluate the load of available gateways without inserting extra overhead, we use the variance in arrival times of periodical broadcasted advertisements. These advertisements can be router advertisements [9] (available in IP version 4 (IPv4) and IP version 6 (IPv6)) or agent advertisements in MIP version 4 (MIPv4). In MIP version 6 (MIPv6), the router advertisement in IPv6 is used. With increased traffic, the gateway may not cope with in-coming and outgoing traffic. This will lead to buffering of advertisements and collisions between advertisements and traffic. If the "Send buffer" at a gateway is full, some advertisements will be dropped. When the link becomes less congested two or more advertisements could be sent in more dense succession. This, in turn, means that with increased traffic the arrival times of advertisements at MHs will vary. Collision of advertisements render in lost advertisements due to broadcast transmission. The metric used is the RVM which is defined by formula 1 and 2 . For a more detailed description, see [5] and [6].

Formula 1 calculates the mean value of the time between arrivals of advertisements and is based on the formula for weighted mean $\left(\bar{x}_{n}\right)$ value [10]. Formula 2 then calculates the variance $\left(V_{n}\right)$ of the arrived advertisements and this is used for the evaluation of wireless links. The variable $t_{n}$ is the arrival time of the last advertisement, $\mathrm{t}_{\mathrm{n}-1}$ is the arrival time of the previous advertisement. The variable $n$ symbolizes the number of advertisements received since the $\mathrm{MH}$ started to receive advertisements from an AP/gateway. With the variable $h$ we select a history window expressing how long history to consider when calculating the mean value and variance.

$$
\begin{aligned}
& \bar{x}_{n}=\frac{1}{h} x_{n}+\frac{h-1}{h} \bar{x}_{n-1} \\
& V_{n}=\frac{1}{h}\left(x_{n}-\bar{x}_{n}\right)^{2}+\frac{h-1}{h} * V_{n-1}
\end{aligned}
$$

The variables $h, \bar{x}_{0}$ and $V_{0}$ are initialized with the following values:

$\frac{1}{h} \in(0,1]$

where $(0,1]$ is the half open interval $\{x: 0<x \leq 1\}$

$V_{0}=0$

$\bar{x}_{0}=$ Defined advertisement time

The variable $\mathrm{x}_{\mathrm{n}}$ is calculated as:

$x_{n}=t_{n}-t_{n-1}$ where $n$ is a integer $>0$

When registered with gateways, the $\mathrm{MH}$ could improve the selection to also include the path in the wired network. We use the round trip time between an $\mathrm{MH}$ and its peer for evaluation of the wired path without inserting extra overhead. The RTT from MIP registration request/reply between the $\mathrm{MH}$ and the HA is added to the RVM value. This metric is named the Relative Network Load (RNL), see formula 3 and 4.

$$
\bar{x}_{n}=\frac{1}{h} x_{n}+\frac{h-1}{h} \bar{x}_{n-1}
$$

where $n$ symbolizes the n:th RTT measurement and $\bar{x}_{n}$ is the weighted mean value

$$
R N L_{n}=\bar{x}_{n}+V_{n}
$$

where $V_{n}$ symbolizes the RVM value 
$\frac{1}{h} \in(0,1]$ where $(0,1]$ is the half open interval

$\{x: 0<x \leq 1\}$

$\bar{x}_{0}=$ is set to the first RTT measurement

Our approach to global connectivity is a combination of proactive and reactive approaches. Connectivity to gateways is proactive and continuously maintained by agent advertisements. The importance of maintaining gateway connectivity is based on the assumption of small ad hoc networks with the same traffic characteristics as in wired IP subnets. Here the major part of the traffic is to $\mathrm{CHs}$ outside the local network. Connectivity between peers within the ad hoc network is reactive. According to the MIP specification, agent advertisements are to be sent "link local". Since we consider ad hoc networks as subnetworks, the advertisements are modified to be sent via multiple hops. The same agent advertisement may then arrive through multiple paths to an $\mathrm{MH}$. The decision of which gateway to use is based on the RVM and RNL. When using the RVM to select gateways to register with, each MH keeps an array consisting of \{gateway-address, last-hop, RVM . The reason for maintaining the last hop is explained by the scenario drawn in figure 1.

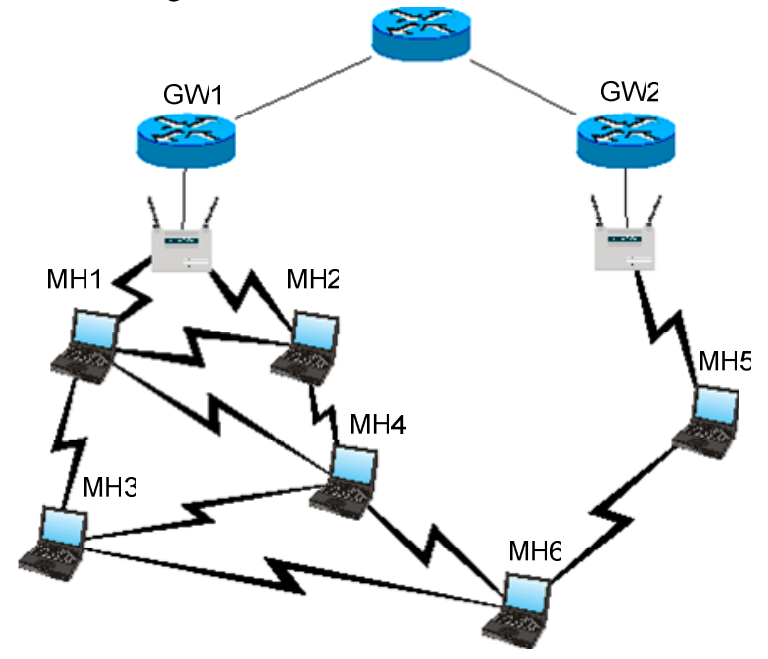

Fig. 1. Topology where MHs calculate the RVM.

If we only uses \{gateway-address, RVM \} as the information to select the gateway (GW1 or GW2), GW1 may be selected in favor of GW2, even though paths to GW1 is more congested by other traffic. The computed RVM may based on advertisements from GW1 giving a lower value than the one computed from GW2. The reason is that there are four nodes (MH1 to MH4) that are able to relay the advertisements and the
MH relaying differ from advertisement to advertisement. While for a route between GW1 and MH6 only one of those nodes will be used. So the RVM does not reflect the load of a single path from GW1 to MH6. By adding the last hop address to the information maintained for a gateway, the RVM can be monitored for each path between GW1 and MH6.

The selection of which agent advertisements to rebroadcast is based on the RVM. The agent advertisement from a previous hop giving the lowest metric for a gateway is rebroadcasted. Figure 2 shows a scenario where there are two gateways (GW1 and GW2) sending agent advertisements. MH1 and MH2 receive advertisements directly from GW1 and via MH3 from GW2. MH3 receives agent advertisements from GW1 via MH1 and MH2 and directly from GW2. MH3 then selects the advertisements with the lowest RVM for each gateway and rebroadcasts these advertisements. In figure 2 this will be the advertisements through $\mathrm{MH} 1$ and the advertisements from GW2.

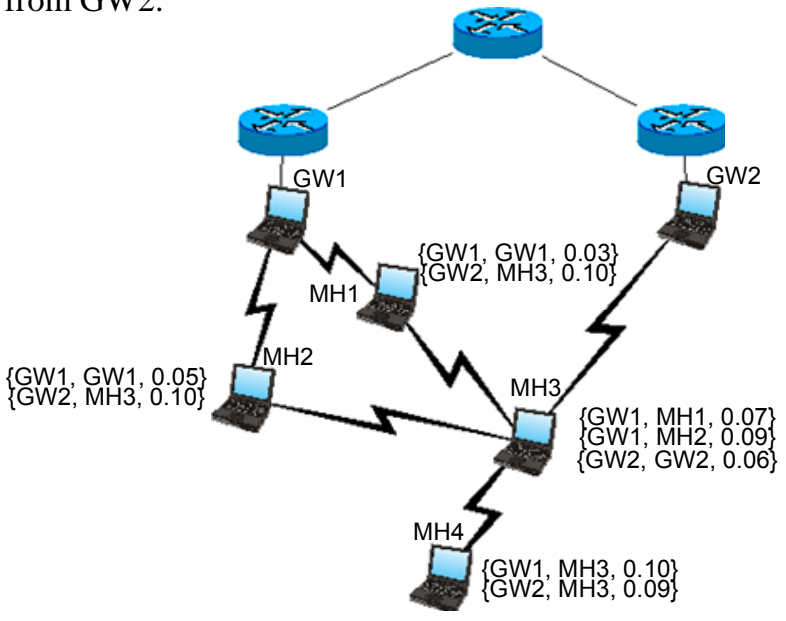

Fig. 2. A scenario showing the propagation of gateway information.

The reason for rebroadcasting advertisements from both gateways is to enable an $\mathrm{MH}$ to register multiple care-of addresses at the HA as well as using route optimization with CHs. Since our proposal only considers small ad hoc networks this is feasible. Figure 3 shows a scenario with a node (MH4) visiting foreign networks. MH4 receives agent advertisements from both gateways. The gateway used for the HA will be set as the default gateway. If MH4 in figure 3 discovers that the route to GW2 is the best route, this care-of address is used to communicate with the HA and hence is selected as the default gateway. The functionality of default routes in currently implemented routing tables assumes the default gateway to be of one hop distance. This means that if MH4 decides to use GW2 in figure 
4, MH4 will have MH3's IP address (130.240.10.110) configured as the default gateway. At the time MH4 makes its decision, MH3 will also have the lowest RVM value to GW2. When MH4 starts to send traffic through GW2 the RVM value in MH3 for GW2 may increase to a value higher than the RVM value calculated for GW1. As defined earlier, a gateway should not be changed while traffic is sent through it in order to avoid flapping between gateways. This means that MH4 should not change gateway until it stops communicating with the peer for a specified period of time or in case the connection to the gateway is lost.

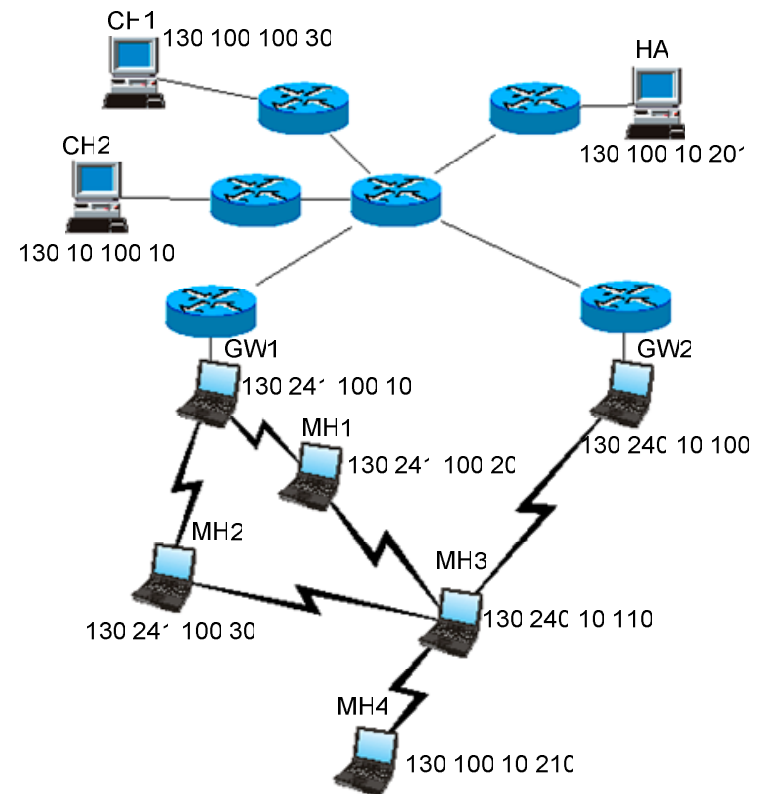

Fig. 3. A topology creating the routing table in figure 4.

If MH3 is not sending or receiving any traffic it is free to select a new gateway. If the RVM value for GW2 increases beyond the RVM for GW1, MH3 selects GW1 as its default gateway and the traffic sent by MH4 will be rerouted to GW1. To avoid this and to make an $\mathrm{MH}$ aware of which gateway it uses, tunneling to the selected gateway is required. This approach differs from the one given in [11] in that the $\mathrm{MH}$ uses the default gateway registered with it's HA when sending packets to a peer (if route optimization is not used). In [11], the functionality of the reactive ad hoc routing protocol was sustained by the $\mathrm{MH}$ sending a route request for all destinations regardless of the destination's IP address. However, with that approach a gateway not associated to the $\mathrm{MH}$ may respond. In the case of reverse tunneling between the FA and the HA to avoid ingress filtering it is required that the $\mathrm{MH}$ uses one of the gateways registered at the HA. Also, since
RVM is used to decide the path to a gateway it should be used both for packets sent and received by the MH.

The routing table created in MH4 for the scenario in figure 3 is shown in figure 4. MH4 uses GW2 as its default gateway. GW1 is selected for communication to $\mathrm{CH} 1$ and $\mathrm{GW} 2$ is used to communicate with $\mathrm{CH} 2$.

To enable tunneling, virtual interfaces are used. In figure 4 , the virtual interface 0 is the interface managing tunneling to GW2 and virtual interface 1 manages the tunnel to GW1. When a packet is sent to a virtual interface, an outer IP header is added to the packet. If $\mathrm{MH} 4$ sends packets to $\mathrm{CH} 1$ in figure 3 there will be two iterations in the routing table. In the first iteration, the forwarding process identifies the destination address 130.100.100.30 and sends the packets to the virtual interface1. This interface is a process that adds an outer header to the packet. The IP address in the outer header will be the address of GW1, i.e., 130.241.100.10. Now the packet is returned to the forwarding process for a second iteration. This time the entry 130.241 .100 .10 is selected. The packet will then be sent to interface 130.100.10.210 with 130.240.10.100 as the next destination.

\begin{tabular}{|l|l|l|l|l|}
\hline Address & Mask & Next hop & Interface & $\begin{array}{l}\text { Metri } \\
\text { c }\end{array}$ \\
\hline 130.10 .100 .10 & $255 \ldots 255$ & Virtual int. 0 & Virtual int. 0 & - \\
\hline 130.100 .100 .30 & $255 \ldots 255$ & Virtual int. 1 & Virtual int. 1 & - \\
\hline 130.241 .100 .10 & $255 \ldots 255$ & 130.240 .10 .110 & 130.100 .10 .210 & 3 \\
\hline 130.240 .10 .100 & $255 \ldots 255$ & 130.240 .10 .110 & 130.100 .10 .210 & 2 \\
\hline 0.0 .0 .0 & 0.0 .0 .0 & 130.240 .10 .110 & Virtual int. 0 & - \\
\hline
\end{tabular}

Fig. 4. The routing table created in $\mathrm{MH} 4$ in figure 3.

The registration request message carries the RNL metric as described in [7] and the decision of which care-of address to use is based on this metric. An $\mathrm{MH}$ communicating with a $\mathrm{CH}$ that has the same network number as the gateway the $\mathrm{MH}$ is connected to uses AODV to discover the route. If the $\mathrm{CH}$ has moved to another network the HA will respond to the route request with a route reply. The packets will be sent to the HA that tunnels them to the CH's current location. If the $\mathrm{CH}$ has a network number that differs from the network where the $\mathrm{MH}$ is connected, the packets will be sent to the default gateway using the maintained route based on agent advertisements. If the default gateway running the $\mathrm{FA}$ has the $\mathrm{CH}$ registered as a visitor in the network, an Internet Control Message Protocol (ICMP) [12] redirect is returned to the $\mathrm{MH}$. The $\mathrm{MH}$ will then request a route to the $\mathrm{CH}$ using AODV. If the $\mathrm{CH}$ is outside the network, the gateway will forward the packets according to the IP routing protocol in the wired IP network. 
When selecting a gateway and starting to send packets, the gateway selection for CH's may not change until any of the following occurs:

- An agent advertisement is lost from the selected gateway, and the RVM computed for some other gateway has becomes lower than the RVM of the selected gateway at the time the selection was made.

- The MH stops sending and receiving packets from the $\mathrm{CH}$ for a specified period of time.

- The network layer connection is considered lost due to three successive lost agent advertisements as defined by MIP.

To maintain routes to gateways and to be able to manage MIP messages without enforcing new broadcasts, the active time out time in AODV is set to the registration timeout in MIP. The period of time a route remains active without being used is in AODV called the active route timeout. A route not used within this time is erased. Agent advertisements are sent once a second and the timeout time for MIP registrations is three times the agent advertisement time (as defined by MIP). This gives a timeout time of MIP registrations of three seconds. This is the same time as the active route timeout proposed in AODV. With these timeout settings a route from an $\mathrm{MH}$ to a gateway is maintained by agent advertisements, registration and binding replies. And a route from a gateway to an $\mathrm{MH}$ is maintained by registration requests and binding updates.

When data is received at the gateway it may operate as an ad hoc node forwarding the data in the ad hoc network or act as a gateway forwarding the packets outside the network. Packets received via a tunnel with the gateway address will be decapsulated and forwarded according to the inner IP header destination field. If the destination is visiting the ad hoc network, the gateway will send an ICMP redirect message to the source. If there is a route for the destination in the gateway, the packets will be sent that route. If the packets are destined for an $\mathrm{MH}$ that has a binding to a foreign network, they will be tunneled to the care-of address. In the case of a packet received without tunneling for a destination homed outside the network and not visiting, an ICMP redirect message is returned to the source and the packets are dropped.

\section{Simulation study}

This section evaluates the usefulness and efficiency of the RNL gateway selection strategy compared to normal hop based selection. Our simulation study uses the GlomoSim simulation model version 2.4 [13]. The simulation area is 2000 by 2000 meters and uses $2 \mathrm{Mbps} 802.11$ radios with a transmission range of 380 meters.

Simulation study results are presented in figures 6, 7 and 8 . The graphs with error bars represent the mean value of multiple simulations (different seeds) using a confidence interval of $95 \%$. Our simulation study has selected the packet-size 512 bytes. Packets about this size are used for example for Voice over IP (VoIP). The advertisements used in the simulations have a size of 32 bytes.

Figure 5 shows the simulation topology. There are two routes the $\mathrm{MH}$ could use to communicate with an Internet node. One route is two hops (GW0) and the other three hops (GW1) in the ad hoc network. There are five pairs of nodes sending traffic in-between them adding to the contention for the medium. The $\mathrm{x}$-axis in the graph shows the number of pairs sending competing traffic (0-5 pairs). The solid line represents RNL selection and the dashed line hop selection. Competing traffic is 25,50 or 71 packets/sec.

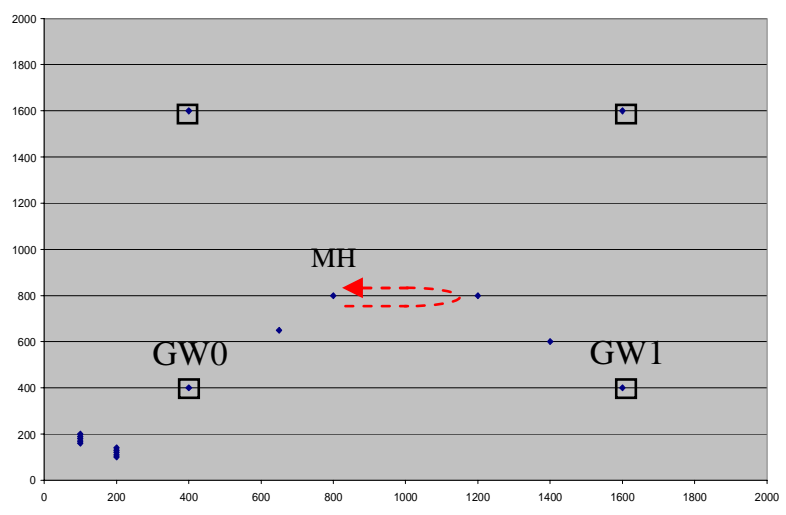

Fig. 5. Simulation topology, $2000^{\star} 2000$ meters.

Figure 6 shows the throughput received at the mobile host. There are five pairs of nodes out of radio range from the $\mathrm{MH}$ but in range of GW0. As they are out of radio range from the MH they will not affect the MHs access to the wireless medium. The movement of the MH would lead to a break in the two hop route. The difference between the algorithms depends on the time spent in an area with several possible routes to the Internet. As expected the effect of RNL selection increases as the number of packets sent increases and as the number of nodes sending traffic increases. 


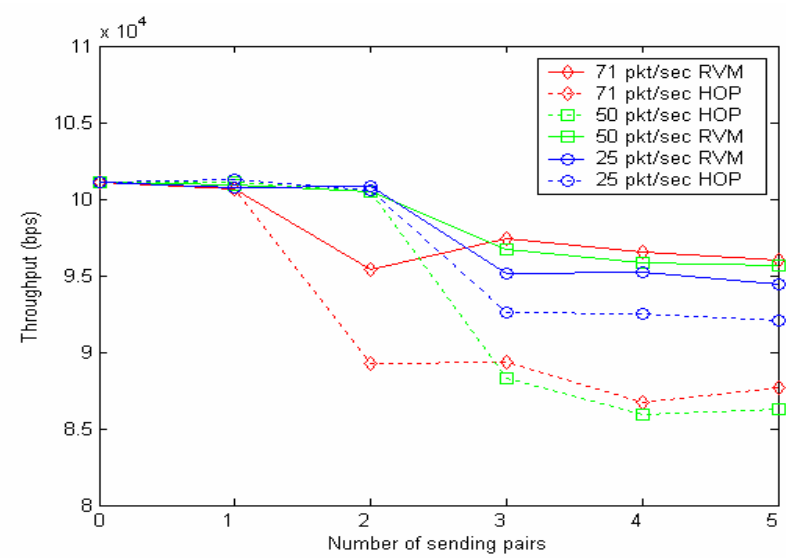

Fig. 6. Throughput received at the $\mathrm{MH}$, competing nodes out of range.

Figure 7 shows the throughput received at $\mathrm{MH}$ when the competing nodes are within radio range (between GW0 and the intermediate node). As seen the contention for the wireless medium sets the limit for the throughput most of the time and the gateway selection only has an effect for a short period of time. As expected the algorithms perform similarly with a small advantage for RNL gateway selection.

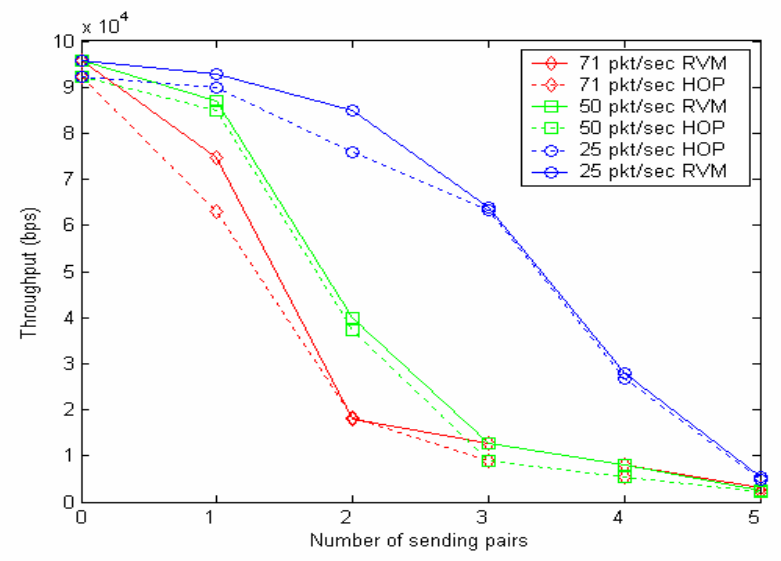

Fig. 7. Throughput received at the $\mathrm{MH}$, competing nodes within range.

Figure 8 shows the throughput received at $\mathrm{MH}$ when the competing nodes and intermediate nodes move with random waypoint $(9.5-10.5 \mathrm{~m} / \mathrm{s}, 2 \mathrm{~s}$ WP-time). Again the gateway selection only has a limited effect since there is not that often there are multiple internet routes available (or even one). As expected the algorithms perform similarly with a small advantage for RNL gateway selection.

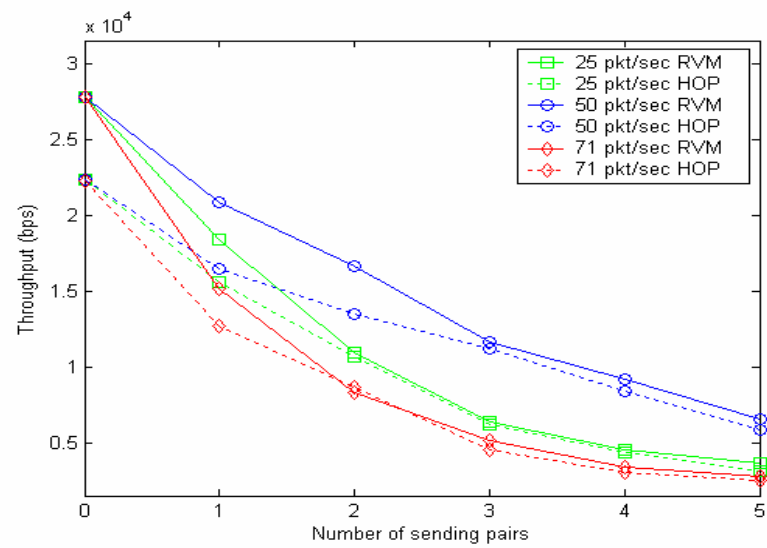

Fig. 8. Throughput received at the $\mathrm{MH}$, nodes random mobility.

\section{Related work}

Belding-Royer, Sun and Perkins [14] propose MIPv4 and AODV be connected so that MIP messages will be managed in the ad hoc network. The question of how to choose between multiple FAs however is not addressed. Moreover an $\mathrm{MH}$ in the ad hoc network has to discover by itself if a destination is within the ad hoc network or not. If the gateway 'thinks' it can reach the destination, it replies with an FA RREP (like the proxy RREP). But before an MH can use the gateway, it first needs to conclude that the destination is not within the ad hoc network and this will delay the connection setup time. Jonsson et al. give in [15] a system description of integration of reactive ad hoc networks and MIP mobility to achieve Internet connectivity. It discusses the benefits of broadcasting MIP advertisements in the ad hoc network and show it is more efficient to use the normal MIP behavior where advertisements are sent without solicitations. Our work extends the benefits of advertisements and introduces a number of improvements of the system. Jonsson et al. [15] and Sun, Belding-Royer and Perkins [16], describe an approach to choosing between multiple FAs. Here the selection is made based on the hop count between the FA and the MH. Hop count may however not be the best way to measure which FA to register with since network load is not considered. A hybrid (proactive/reactive) approach for gateway discovery is proposed by Ratanchandani and Kravetsin [17]. That approach requires nodes to do route discovery for the gateway when sending traffic to Internet destinations. To avoid this delay we insert routes to the gateway making double use of the advertisements. The work uses hop count for gateway selection while we use the advertisement in a third way to calculate network layer 
load as a basis for selection. Nordström et al. describes in [18] the effectiveness of using tunneling as a gateway forwarding strategy, but suffers from the delay introduced by gateway route discovery. It also points out the risk for inconsistent routes if not using gateway tunneling. Lundgren et al. [19] point out the problem with communication gray zones. We do to some extent address this problem by requiring a node to register with its home agent before using a route through a gateway. This imposes unicast packets to be sent in both directions. The support of a wireless backbone and the preference of stable routes are discussed in [20]. The support of a wireless backbone is beneficial in many situations and conforms well to ad hoc access network scenarios. Stable routes are also discussed in [21] and support our recognition of problems with shortest path routes. PULSE [22] is a similar approach to use periodical broadcast to install routes to a pulse source, creating a tree routing structure. Some pulse benefits are continuous route maintenance and power saving functions.

\section{Conclusions}

This paper proposes solutions towards enabling and supporting global connectivity in wireless ad hoc networks. We describe the use of the Running Variance Metric (RVM) [5] and Relative Network Load (RNL) as performance metrics to classify the traffic load of gateways in wireless access networks. A gateway selection strategy and its effect on performance in multi-hop ad hoc networks is evaluated. The gateway selection based on RNL is compared to normal hop based selection. RNL gateway selection is shown to perform better when it is beneficial to switch a short congested route to one gateway to a longer one to another gateway.

The algorithms perform similarly in scenarios where the medium contention has the greatest impact on throughput, when the medium is below congestion or when there is only one gateway available. A mobility scenario is such a scenario when the RNL selection would react quicker and switch over to a longer route before the shorter route breaks.

The simulation study reported in this paper demonstrates that RNL gateway selection, used in ad hoc access networks, enhances the throughput. This simulation study also supports the theoretical contribution presented in [11,23].

A Global Connectivity access network with our solutions manages handover and multihoming. We use RVM with Multihomed MIP as described in [23] to associate with multiple gateways. With the proposed approach it is possible to select the least loaded gateway(s) while doing handover when two or more gateways is used. No double casting or multicasting is needed because the $\mathrm{MH}$ is connected to multiple gateways receiving unique packets. By this, the functionality at the data-link layer is sustained. Multiple associations are maintained in order to evaluate the performance of gateways.

In future work we will perform more evaluation of other traffic patterns and mobility scenarios.

\section{References}

[1] M. S. Gast. 802.11 Wireless Networks, The Definite Guide, O'Reilly, 2002.

[2] U. Varshney, The status and future of 802.11-based WLANs Computer, vol. 36, no. 6, pp. 102-105, Jun, 2003.

[3] C. Eklund, R. B. MArks, and K. L. Stanwood, IEEE Standard: 802.16 A Technical Overview of the WirelessMAN Air Interface for Broadband Wireless Access IEEE Communications Magazine, vol. 40, no. 6, pp. 98107, Jun, 2002.

[4] Cisco Systems. NBMA, http://searchnetworking.techtarget.com/Definition/0,,sid7_gc i838049,00.html. 2003.

[5] C. Ahlund, R. Brannstrom, and A. Zaslavsky, "Running Variance Metric for evaluating performance of Wireless IP Networks in the MobileCity Testbed," International Conference on Testbeds and Research Infrastructures for the Delevopment of Networks and Communities, Feb. 2005.

[6] C. Perkins, Mobile IP IEEE Communications Magazine, vol. 40, no. 5, pp. 66-82, May, 2002.

[7] C. Ahlund, R. Brannstrom, and A. Zaslavsky, "M-MIP: extended Mobile IP to maintain multiple connections to overlapping wireless access networks," International Conference on Networking, Apr. 2005.

[8] C. E. Perkins and E. M. Belding-Royer, "Ad-hoc On Demand Distance Vector Routing," 2nd IEEE Workshop on Mobile Computing Systems and Applications, pp. 90-100, Feb. 1999.

[9] S. Deering. ICMP Router Discovery Message, IETF RFC 1256. 91.

[10] L. Rade and B. Westergren. Beta Mathematics Handbook, Studentlitteratur,

[11] C. Ahlund and A. Zaslavsky, Extending Global IP Connectivity for Ad Hoc Networks Telecommunication Systems, Modeling, Analysis, Design and Management, vol. 24, no. 2, pp. 221-250, Oct, 2003. 
[12] J. Postel. Internet Control Message Protocol, IETF RFC 792. 81.

[13] UCLA Parallel Computing Laboratory. Glomosim, http://pcl.cs.ucla.edu/projects/glomosim/. 2004.

[14] E. M. Belding-Royer, Y. Sun, and C. E. Perkins. Global connectivity for IPv4 mobile ad hoc networks, Internet Draft. 2001.

[15] U. Jönsson, f. Alriksson, T. Larsson, P. Johansson, and G.-Q. Maguire, "MIPMANET-Mobile IP for Mobile Ad Hoc Networks," International Symposium on Mobile Ad Hoc Networking \& Computing, pp. 75-85, Aug. 2000.

[16] Y. Sun, E. M. Belding-Royer, and C. E. Perkins, Internet Connectivity for Ad hoc Mobile Networks International Journal of Wireless Information Networks special issue on 'Mobile ad Hoc Networks (MANETs): Standards, esearch, Applications', vol. 9, no. 2, pp. 75-88, Apr, 2002.

[17] P. Ratanchandani and R. Kravets, "A Hybrid Approach to Internet Connectivity for Mobile Ad Hoc Networks," Wireless Communications and Networking, pp. 1522-1527, Mar. 2003.

[18] E. Nordström, P. Gunningberg, and C. Tschudin, "Gateway Forwarding Strategies for Ad hoc Networks," 4th Scandinavian Workshop on Wireless Ad hoc Networks , May 2004.

[19] H. Lundgren , E. Nordström, and C. Tschudin, "Coping with Communication Gray Zones in IEEE 802.11b based Ad hoc Networks," 5th ACM international workshop on Wireless mobile multimedia, 2002.

[20] J. Shin, J. Na, H. Lee, A. Park, and S. Kim, "Mobile IP Support in Ad Hoc Networks with Wireless Backbone," IEEE 59th Vehicular Technology Conference, pp. 21362139.

[21] E. Curran and J. Dowling, "SAMPLE: Statistical Network Link Modelling in an On-Demand Probabilistic Routing Protocol for Ad Hoc Networks," Second Annual Conference on Wireless On demand Network Systems and Services, 2005.

[22] B. Awerbuch, D. Holmer, and H. Rubens, "The Pulse Protocol: Mobile Ad hoc Network Performance Evaluation," Second Annual Conference on Wireless On-demand Network Systems and Services, Jan. 2005.

[23] C. Ahlund and A. Zaslavsky, "Multihoming with Mobile IP," 6th IEEE International Conference on High Speed Networks and Multimedia Communications, pp. 235-243, July 2003. 